\title{
Interactive comment on "Stratospheric Variability at a glance - Analysis of the intra decadal timescale and the QBO" by Duy Cai et al.
}

\section{Anonymous Referee \#1}

Received and published: 23 November 2016

This paper analyzes an intradecadal spectrum of the stratosphere using a reanalysis and compares it with model simulations. One of the main differences in the intradecadal variability between the reanalysis and model results is associated with the QBO in the tropics. The authors assessed simulated equatorial waves in intraseasonal spectra of the middle stratosphere, and attributed the defect in simulating the QBO to underrepresentation of the equatorial waves in the models.

Major comments :

The above findings, however, have been well known by previous studies, as the authors also mentioned in the paper. The authors did not extend their analysis further, and thus the paper does not seem to provide concrete ideas that can support or add on 
our understanding on this topic. Based on the Introduction section, this paper seems motivated by the potential impacts of model-representation of the stratospheric variability upon the (intra) decadal predictability. I agree that this is very interesting topic. However, it might be not easy to address/assess the potential impacts of the stratospheric variability using this relatively short-term (20 years) climate simulation. In addition, by estimating the typical vertical scales of the equatorial waves presented in the reanalysis, the authors discussed the model vertical resolution that is required to simulate the QBO $(<1 \mathrm{~km})$. Regarding this, readers may expect more contents than those presented here (e.g., actual spatiotemporal structures of waves and their propagation in simulations using different stratospheric resolutions) because many recent papers have demonstrated this point even in more details (e.g., Krismer and Giorgetta, 2014; Richter et al., 2014; Anstey et al., 2016). Considering the contents of the paper, I would not recommend to publish this manuscript in ACP.

\section{Specific comments :}

The manuscript requires linguistic advice in terms of sentence structures, use of comma, etc.

L16: "are" $\rightarrow$ "is"

L21: This sentence is not relevant to this paragraph. Please move it to $L 43$.

L26: "showed that"

L41: "QBO and in" $\rightarrow$ "QBO. In"

L42: Scaife et al. (2014) showed that the skillful prediction of the QBO solely does not guarantee the predictability of the winter surface.

L48: "final section 5" $\rightarrow$ "final section"

L58: What does the "low-level" mean ? Vertical level ? Or, level of components ? 
Please clarify it.

L65: Please provide the full name for IPCC.

L66-67: Please delete this sentence.

L69: Please insert the comma after "spin-up".

L73: Is this the 'effective' resolution? What is the vertical grid spacing here?

Interactive

comment

L81: Please place this reference to L53.

L86: "squares" $\rightarrow$ "square"

L95: Delete "and a 6 months".

L100: Please provide the full name for FFT.

L100-101: This is an ordinary way to get the variances. Please delete this sentence.

L102: If you would sum over all wavelengths, you did not have to perform the 2dimensional FFT for the decadal time series.

L115 and L259: I suspect that the paper by Lindzen and Holton (1968) might be not a proper reference here. I guess that you intended to refer to Lindzen and Matsuno (1968). Please confirm these.

L120: "n = 1" $\rightarrow$ "n = -1"

L121: "number is" $\rightarrow$ "number we consider is"

L130-137: This description does not seem really necessary.

L140 and Fig. 1 caption: The log of any variables cannot have a unit (unless you specifically define the quantity with a reference power, like decibel [dB]).

L140-141: So obvious statement. Please remove it.

L142: "variability" $\rightarrow$ "power" 
L143: Delete "in particular".

L145: Please rephrase "polar vortex contribution to the variability" to clarify the meaning.

L147: Include "for periods shorter than 6 months" after "latitudes"

L154: "provide"

Interactive

comment

L153-158: This fact is very well known. The authors could just add some references here rather than describe this in detail. In addition, Fig. 2 does not address more than what we know.

L165: “... hemisphere high latitudes”

L166: "intra-annual and intra-seasonal": What are the difference?

L172: "The QBO comes close to exhibiting ...": Please make the sentence clear.

L173: Delete "about the equator".

L188-189: Please move this sentence to Section 2.

L189: Delete "this".

L199: Synoptic waves may have zonal wavenumber larger than 4 ( $k=4$ corresponds to the wavelength of $10000 \mathrm{~km}$ in the tropics). Please delete "and synoptic".

L208: "0.1" $\rightarrow$ "0.03"

L230: Delete "pattern and" unless the "pattern" here has a different meaning from the "shape" and you will describe both.

L239-241: I could not understand this sentence. Please rewrite it.

L244: "30" $\rightarrow$ "3" (or 2 days?)

L258: "linear equatorial wave theory was" 
L266: Note that the equation for $\mathrm{N}^{2}$ written here is correct only if $\mathrm{H}$ is defined as $\mathrm{RT} / \mathrm{g}$, where $\mathrm{T}$ is a mean temperature that actually represents the temperature in the tropics at this altitude (Eq. 1.1.13 on p6 in Andrews et al., 1987).

L269: "Figure 6"

L269: How did you obtain the climatological mean of the vertical wavelengths of MRG waves? It is spectral-power-weighted mean?

L280: "corresponding" $\rightarrow$ "those of"

L285-286: Please rephrase the sentence.

L289: "to" $\rightarrow$ "and"

L300: "simulations appear."

L304: "frequency-wavenumber"

L318: Please delete "(which are the main drivers)" because it is believed that the IG and mesoscale gravity waves may also provide large momentum, as well as the Kelvin waves.

L331: I do not agree that this paper shows it.

L336-338: This is a rather unexpected statement because there was no discussion regarding the gravity wave parameterization before this.

\section{References:}

Andrews, D. G., Holton, J. R., and Leovy, C. B.: Middle atmosphere dynamics, 489 pp., Academic, San Diego, Calif, 1987.

Anstey, J. A., Scinocca, J. F., and Keller, M.: Simulating the QBO in an atmospheric general circulation model: Sensitivity to resolved and parameterized forcing, J. Atmos. Sci., 73, 1649-1665, 2016. 
Krismer, T. R. and Giorgetta, M. A.: Wave forcing of the quasi-biennial oscillation in the Max Planck Institute Earth System Model, J. Atmos. Sci., 71, 1985-2006, 2014.

Richter, J. H., Solomon, A., and Bacmeister, J. T.: On the simulation of the quasibiennial oscillation in the Community Atmosphere Model, version 5, J. Geophys. Res. Atmos., 119, 3045-3062, 2014.

Interactive

comment

Interactive comment on Atmos. Chem. Phys. Discuss., doi:10.5194/acp-2016-870, 2016. 